\title{
The dynamics of a levitated cylindrical permanent magnet above a superconductor
}

\author{
Michael SCHREINER \\ Interstate University of Applied Sciences of Technology \\ Laboratory for Industrial Mathematics \\ Werdenbergstrasse 4 \\ CH-9471 Buchs, Switzerland \\ schreiner@ntb.ch
}

Recibido: 28 de Abril de 2003

Aceptado: 17 de Julio de 2003

\begin{abstract}
When a permanent magnet is released above a superconductor, it is levitated. This is due to the Meissner-effect, i.e. the repulsion of external magnetic fields within the superconductor. In experiments, an interesting behavior of the levitated magnet can be observed: it might start to oscillate with increasing amplitude and some magnets even reach a continuous rotation. In this paper we develop a mathematical model for this effect and identify by analytical methods as well with finite element simulations two reasons for the described behavior of the levitated magnet. It is shown, that the oscillations of the magnet are due to inhomogeneities of the magnetization within the magnet, whereas the continuous rotations are due to temperature gradients of the surrounding air.
\end{abstract}

2000 Mathematics Subject Classification: 82D55, 35K05, 37N20, 74H55.

Key words: Superconductor, Meissner effect, levitated permanent magnet, rotating permanent magnet

\section{Introduction}

Soon after the discovering of high temperature superconductivity (cf. [1]), it was found that a permanent magnet is levitated above a high temperature superconductor and rests there in a stable position (cf. [2]). This effect is due to the so-called Meissner repulsion of external magnetic fields within the superconductor. In other words, the external magnetic field induces currents in the superconductor, which produce a second magnetic field, which lifts up the magnet (cf. e.g. [9]). 


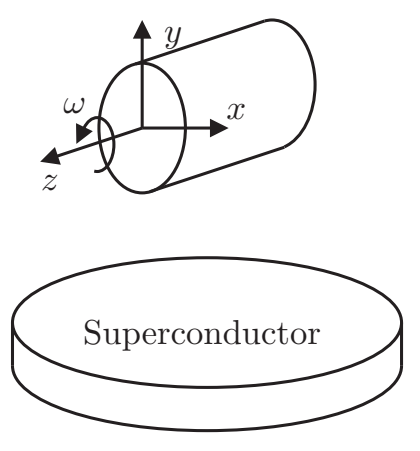

Figure 1: The experimental setup. Above a superconductor, there is a levitated cylindrical permanent magnet.

In Figure 1, a typical experimental setup is sketched. A cylindrical rare earth compound magnet which is magnetized along its axis, is levitated above a cooled high $T_{c}$-superconducting disk made of $\mathrm{YBa}_{2} \mathrm{Cu}_{3} \mathrm{O}_{7}$.

Experiments show that the cylindrical magnet starts to oscillate around its horizontal axis with increasing amplitude and will eventually rotate continuously with a nearly constant angular speed. The effect was explained independently in [5], [7], and [10] (see also [9]). The energy for the rotation is due to temperature differences of the surrounding air. Thus, the described effect represents a miniature heat engine.

In this paper, we develop a model for the system, which is an extension of the model described in [11]. With a detailed analysis it is possible to identify two sources for the rotating behavior of the permanent magnet. There is a driving torque which is caused by the temperature gradient of the surrounding air and depends on the velocity. For a stationary solution it is possible to give an explicit expression for this torque. It depends on the angular velocity $\omega$ and the Fourier coefficients of the temperature of the surrounding air of order one. A second source is a restoring torque which is due to inhomogeneities of the magnetization of the permanent magnet. Ignoring the temperature gradient, the governing differential equation for the permanent magnet is that of a mathematical pendulum.

The outline of the paper is as follows: We start with the development of the model. After that we consider two idealizations: The perfect permanent magnet (i.e. the magnetization is homogeneous), and a homogeneous temperature field of the surrounding air. After that, we present the results of finite element simulations. We finish the paper with some conclusions. 


\section{The Model}

In this chapter, a detailed model for the levitated permanent is developed. We start with the geometric setting in three dimensions and its idealization in two dimensions. The energy for the rotation is based on the surrounding temperature field. Thus, the heat transfer is described next. For the later considerations it is of advantage to have a closer look at the time behavior of the mean temperature within the permanent magnet. After that, the magnetic forces and the resulting torque are modelled. It turns out that there are two sources for the torque acting on the magnet: First, the dependence of the magnetization of the permanent together with temperature gradients of the surrounding air yield a torque $M_{T}$. Second, the inhomogeneous magnetization of the permanent magnet (independent of the temperature) produces a restoring torque $M_{\varphi}$. The equations of motion are formulated next, and then we finally present the whole system of coupled partial differential equations.

\subsection{Geometry}

The rare earth compound magnet has a cylindrical shape $V$ with length $l$ and radius a. The magnet is levitated above a superconducting disk (cf. Figure 1). From experiments we know, that the changes in the height are negligible, so that we can choose a fixed coordinate system where the $z$-axis coincides with the axis of the magnet. The magnetization is in the direction of the $z$-axis. We assume a constant density $\rho$ of the magnet, so that the total mass is $\pi a^{2} l \rho$.

It is possible to treat parts of the problem in a two-dimensional setting. In these cases, we will look at a cross section of the cylinder, i.e. the circle $A$ in the $x y$-plane with radius $a$ and center $(0,0)$. The normal vector at a point of the boundary $\partial A$ is denoted by $\mathbf{n}$, and assumed to point into the surrounding air.

The superconducting plane is parallel to the $x z$-plane and has a negative $y$ coordinate, so that the levitation forces acting on the permanent magnet point into positive $y$-direction.

\subsection{Heat Transfer}

Assuming that the temperature in $V$ does not change in $z$-direction and that the heat flux through the bottom and top face of the cylinder is negligible, it is sufficient to consider the heat transfer in the circle $A$. If we ignore for a moment the rotation of the magnet, the temperature distribution $T=T(t, x, y)$ is governed by the twodimensional time dependent heat equation (cf. [3])

$$
\rho c \frac{\partial T}{\partial t}-\nabla \cdot(\lambda \nabla T)=0
$$

The right hand side is zero since there are no heat sources. $c$ is the specific heat and $\lambda$ the thermal conductivity. This equation has to be completed with an initial condition 
$T(0, x, y)=T_{0}(x, y)$ and boundary conditions. We apply Newtons law of cooling, i.e. at the boundary $\partial A$ we assume a heat flux

$$
\mathbf{n} \cdot(\lambda \nabla T)=\alpha\left(T_{\text {sur }}-T\right)
$$

where the temperature $T_{\mathrm{Sur}}=T_{\mathrm{Sur}}(x, y)$ of the surrounding air is constant in time and known from measurements. When appropriate, we sometimes write $T_{\mathrm{sur}}(\varphi)$ for $T_{\operatorname{sur}}(a \cos \varphi, a \sin \varphi) . \alpha$ is the heat transfer coefficient.

If the permanent magnet rotates with angular speed $\omega(\mathrm{rad} / \mathrm{s})$, we have to change the heat equation accordingly. We take the Eulerian point of view, i.e. we assume the coordinate system to be fixed and not rotating with the magnet. Then we have to take into account a convective heat transfer in the disk $A$. The point $(x, y)$ moves with the velocity $\omega(-y, x)^{T}$, so that we complete equation (1) with a convection term:

$$
\rho c\left[\frac{\partial T}{\partial t}+\omega\left(\begin{array}{c}
-y \\
x
\end{array}\right) \cdot \nabla T\right]-\nabla \cdot(\lambda \nabla T)=0 .
$$

Next, we study the time evolution of the mean temperature in the permanent magnet, i.e.

$$
\bar{T}(t)=\frac{1}{\pi a^{2}} \int_{A} T(t, x, y) d A
$$

Theorem 2.1. Let $T=T(t, x, y)$ be the solution of (2) and (3) with respect to an arbitrary initial condition. Then it holds for the mean temperature (4) of the permanent magnet

$$
\lim _{t \rightarrow \infty} \bar{T}(t)=\bar{T}^{\infty}
$$

with

$$
\bar{T}^{\infty}=\frac{1}{2 \pi} \int_{0}^{2 \pi} T_{s u r}(\varphi) d \varphi .
$$

The limit is independent of $\omega$ and thus uniformly with respect to $\omega$.

Proof. In polar coordinates $(x=r \cos \varphi, y=r \sin \varphi$ ), equation (3) reads as

$$
\frac{\partial T}{\partial t}+\omega \frac{\partial T}{\partial \varphi}-\lambda\left(\frac{\partial^{2}}{\partial r^{2}}+\frac{1}{r} \frac{\partial}{\partial r}\right) T-\frac{\lambda}{r^{2}} \frac{\partial^{2}}{\partial \varphi^{2}} T=0 .
$$

We integrate this equation according to $\int_{0}^{2 \pi} \ldots d \varphi$. For smooth solutions, the order of differentiation and integration can be interchanged, and we get

$$
\begin{gathered}
\frac{\partial}{\partial t} \int_{0}^{2 \pi} T(t, r, \varphi) d \varphi+\omega \int_{0}^{2 \pi} \frac{\partial}{\partial \varphi} T(t, r, \varphi) d \varphi \\
-\lambda\left(\frac{\partial^{2}}{\partial r^{2}}+\frac{1}{r} \frac{\partial}{\partial r}\right) \int_{0}^{2 \pi} T(t, r, \varphi) d \varphi-\frac{\lambda}{r^{2}} \int_{0}^{2 \pi} \frac{\partial^{2}}{\partial \varphi^{2}} T(t, r, \varphi) d \varphi=0 .
\end{gathered}
$$


The integrals with partial derivatives with respect to $\varphi$ vanish, so that the abbreviation

$$
\tau(t, r)=\int_{0}^{2 \pi} T(t, r, \varphi) d \varphi
$$

yields the weakly singular differential equation

$$
\frac{\partial}{\partial t} \tau(t, r)-\lambda\left(\frac{\partial^{2}}{\partial r^{2}}+\frac{1}{r} \frac{\partial}{\partial r}\right) \tau(t, r)=0 \text { for } r \in(0, a) .
$$

For the boundary condition we get similarly

$$
h \frac{\partial \tau}{\partial r}+\left.\tau\right|_{r=a}=\int_{0}^{2 \pi} T_{\operatorname{Sur}}(\varphi) d \varphi .
$$

Thus, $\tau(t, r)$ is the solution of a rotational symmetric heat transfer equation (7) corresponding to the boundary condition (8). For the solution it is known (cf. [12]), that

$$
\lim _{t \rightarrow \infty} \tau(t, r)=\mathrm{const}=\int_{0}^{2 \pi} T_{\mathrm{Sur}}(\varphi) d \varphi
$$

Since

$$
\bar{T}(t)=\frac{1}{\pi a^{2}} \int_{0}^{a} r \tau(t, r) d r
$$

it follows

$$
\lim _{t \rightarrow \infty} \bar{T}(t)=\frac{1}{\pi a^{2}} \frac{1}{2} a^{2} \int_{0}^{2 \pi} T_{\operatorname{sur}}(\varphi) d \varphi=\bar{T}^{\infty},
$$

as required. Since $\omega$ has canceled out during the calculations, the limit is uniformly with respect to $\omega$.

We have seen that the mean temperature in the cylindrical magnet converges to $\bar{T}^{\infty}$. It will be of advantage, to linearize the temperature dependency of the magnetization of the permanent magnet around this temperature. This will be done in the next section.

\subsection{Magnetic Forces}

In this section we look at the forces acting on the permanent magnet, and at the torque, which drives the rotation of the magnet.

Since the permanent magnet is levitated above the superconductor, the total levitation force is obvious. It holds

$$
\mathbf{F}=\left(\begin{array}{c}
0 \\
\pi r^{2} l \rho g \\
0
\end{array}\right)
$$


In order to understand what is causing the moments on the permanent magnet, we have to take a deeper look at the magnetic forces: We assume that the permanent magnet consists out of a volume density $\mathbf{m}$ of magnetic dipoles. If $\mathbf{B}^{0}$ is the magnetic field due to the induced currents in the superconducting plate, then (cf. e.g. [8]) the force on the permanent magnet is given by

$$
\mathbf{F}=\int_{V} \nabla\left(\mathbf{m} \cdot \mathbf{B}^{0}\right) d V=\int_{V}(\mathbf{m} \cdot \nabla) \mathbf{B}^{0} d V
$$

(We assume $\mathbf{m}$ to be constant in $V$.)

Some simplifications are in order. First note that $\mathbf{m}$ only has nonzero components in $z$-direction (that is the axis of the permanent magnet), so that only the $z$-component $B_{z}^{0}$ of $\mathbf{B}^{0}$ has to be considered. If we assume, that $B_{z}^{0}$ is constant in $z$-direction within the small volume $V$, i.e. $B_{z}^{0}(x, y, z)=B_{z}^{0}(x, y)$, we can simplify (10) to

$$
\mathbf{F}=\int_{V} m_{z} \nabla B_{z}^{0} d V=\int_{0}^{l} \int_{A} m_{z} \nabla B_{z}^{0} d A d z=l \int_{A} m_{z} \nabla B_{z}^{0} d A .
$$

Before we start calculating the moments, some linearizations should be introduced: We know from e.g. [8] that $B_{z}^{0}$ is decreasing with $1 / r^{3}$ over the superconductor, when $r$ denotes the distance from the superconductor. Since the diameter of $A$ is small, we may linearize $B_{z}^{0}$ in $A$, i.e. we assume $\nabla B_{z}^{0}$ to be constant in $A$,

$$
\nabla B_{z}^{0}=\left(\begin{array}{c}
0 \\
g_{B} \\
0
\end{array}\right)
$$

with a suitable (negative) constant $g_{B}$. That the $x$ - and $z$-component are zero is due to the fact, that the total force (10) has zero $x$ - and $z$-components.

Thus, the $y$-component of the force (11) is given by

$$
F_{y}=\lg _{B} \int_{A} m_{z}(x, y) d A
$$

where we denote the dependence of the magnetization on the positions explicitly via $m_{z}(x, y)$.

An important reason for the driving torque of the permanent magnet is the temperature dependency of the magnetization. It is known from measurements (cf. e.g. $[5])$, that in the temperature range we are concerned with the magnetization increases, when the temperature decreases. In our application, the temperature differences are small, so that a linear model is appropriate. The magnetization at the limit mean temperature $\bar{T}^{\infty}$ is denoted by $m_{z}^{\infty}(x, y)$, so that the model for the magnetization is

$$
m_{z}(x, y)=m_{z}^{\infty}(x, y)+\frac{\gamma}{\pi a^{2}} \int_{A} m_{z}^{\infty}(\xi, \eta) d \xi d \eta\left(T(x, y)-\bar{T}^{\infty}\right)
$$


As mentioned before, the constant $\gamma$ is negative. By writing the temperature dependence in this way, we have

$$
\frac{m_{z}(x, y)-m_{z}^{\infty}(x, y)}{\frac{1}{\pi a^{2}} \int_{A} m_{z}^{\infty} d A}=\gamma\left(T(x, y)-\bar{T}^{\infty}\right),
$$

so that $\gamma$ is the relative increase of the magnetization, when the temperature change is 1 (Kelvin).

With this model for the magnetization, the force (13) can be written as

$$
F_{y}=l g_{B} \int_{A} m_{z}^{\infty}(x, y) d A+\gamma l g_{B} \int_{A} m_{z}^{\infty}(x, y) d A \frac{1}{\pi a^{2}} \int_{A}\left(T(x, y)-\bar{T}^{\infty}\right) d A
$$

so that

$$
F_{y}=l g_{B} \int_{A} m_{z}^{\infty}(x, y) d A\left[1+\gamma\left(\bar{T}-\bar{T}^{\infty}\right)\right] .
$$

Next, we switch to the torque $M$. Since the force has only components in $y$ direction, we have

$$
\begin{aligned}
M & =l g_{B} \int_{A} x m_{z}(x, y) d A \\
& =l g_{B} \int_{A} x m_{z}^{\infty}(x, y) d A+\gamma l g_{B} \int_{A} m_{z}^{\infty}(x, y) d A \frac{1}{\pi a^{2}} \int_{A} x\left(T(x, y)-\bar{T}^{\infty}\right) d A
\end{aligned}
$$

In the second integral we do not write $x m_{z}^{\infty}(x, y)$, since this integral comes from the ansatz of the linear temperature dependence.

We have to be careful about the meaning of $x$ and $y$ in the previous formula: In

$$
\int_{A} x m_{z}^{\infty}(x, y) d A
$$

$m_{z}^{\infty}(x, y)$ describes the magnetization of the permanent magnet. Thus, when the magnet rotates, $(x, y)$ is meant to be fixed at the rotating coordinate system of the cylinder. The $x$ in front of $T(x, y)$ is the lever arm of the magnetic force and therefore meant to correspond to the fixed coordinate system, where the temperature is described in. Thus, we should change the notation. We assume that the angle of the permanent magnet is denoted by $\varphi$ and that for $\varphi=0$ the global coordinate system and the coordinate system of the magnet coincide. For $\varphi=0$ we introduce the abbreviations

$$
x_{0}^{m}=\frac{\int_{A} x m_{z}^{\infty}(x, y) d A}{\int_{A} m_{z}^{\infty}(x, y) d A}, \quad y_{0}^{m}=\frac{\int_{A} y m_{z}^{\infty}(x, y) d A}{\int_{A} m_{z}^{\infty}(x, y) d A},
$$


so that the point $\left(x_{0}^{m}, y_{0}^{m}\right)$ is the center of the magnetization of the permanent magnet at the angle $\varphi=0$. This point is fixed at the magnet and rotates with it, so that we write for an arbitrary angle $\varphi$

$$
\begin{aligned}
& x_{\varphi}^{m}=x_{0}^{m} \cos \varphi-y_{0}^{m} \sin \varphi \\
& y_{\varphi}^{m}=x_{0}^{m} \sin \varphi+y_{0}^{m} \cos \varphi .
\end{aligned}
$$

Thus, the first term of (16) is recognized to be dependent on the angle $\varphi$ which describes the rotation of the permanent magnet. We therefore write

$$
\begin{aligned}
M_{\varphi} & =\lg _{B} \int_{A} x m_{z}^{\infty}(x, y) d A \\
& =\lg _{B} \int_{A} m_{z}^{\infty}(x, y) d A\left[x_{0}^{m} \cos \varphi-y_{0}^{m} \sin \varphi\right]
\end{aligned}
$$

The second term in (16) describes the part of the torque which is due to the temperature distribution in the cylinder. The local temperature is measured in the global coordinate system (cf. Section 2.2). Therefore, this part of (16) describes the torque due to the temperature field which is independent of the angle $\varphi$. Thus, we write

$$
M_{T}=\gamma \lg _{B} \int_{A} m_{z}^{\infty}(x, y) d A \frac{1}{\pi a^{2}} \int_{A} x T(x, y) d A .
$$

The limit temperature $\bar{T}^{\infty}$ cancels out, since $\int_{A} x \bar{T}^{\infty} d A=\bar{T}^{\infty} \int_{A} x d A=0$ by symmetry.

We have seen that the total torque $M$ can be split into two summands $M=M_{\varphi}+M_{T}$. $M_{\varphi}$ is independent of the temperature and depends only on the magnetization at temperature $\bar{T}^{\infty}$ and the angle $\varphi$ of the permanent magnet. The second term $M_{T}$ is independent of $\varphi$ and is due to the solution $T$ of the heat equation.

Assume for the moment that $\bar{T}=\bar{T}^{\infty}$ (which is the limit for $t \rightarrow \infty$ ). Then it follows from (9) and (14) that

$$
\lg _{B} \int_{A} m_{z}^{\infty}(x, y) d A=\pi r^{2} l \rho g .
$$

We denote this levitation force by $\bar{F}_{y}^{\infty}=\pi r^{2} l \rho g$. Using this abbreviation, we finally arrive at the following equations for the driving torque of the magnet:

$$
\begin{aligned}
M & =M_{\varphi}+M_{T} \\
M_{\varphi} & =\bar{F}_{y}^{\infty}\left[x_{0}^{m} \cos \varphi-y_{0}^{m} \sin \varphi\right] \\
M_{T} & =\bar{F}_{y}^{\infty} \gamma \frac{1}{\pi a^{2}} \int_{A} x T(x, y) d A
\end{aligned}
$$

In other word, we can imagine that the force $\bar{F}_{y}^{\infty}$ has two different lever arms: the $\varphi-$ dependent $\left[x_{0}^{m} \cos \varphi-y_{0}^{m} \sin \varphi\right]$ and the temperature-dependent $\gamma \frac{1}{\pi a^{2}} \int_{A} x T(x, y) d A$. 


\subsection{Equation of Motion}

Now we consider the rotation of the permanent magnet which is due to the driving torque $M=M_{\varphi}+M_{T}$. With the moment of inertia $J=l \pi \rho a^{4} / 2$, we simply have

$$
\begin{gathered}
\omega=\frac{\partial \varphi}{\partial t}, \\
J \frac{\partial \omega}{\partial t}+\mu_{r} \omega=M_{\varphi}+M_{T} .
\end{gathered}
$$

Wa have assumed Stokes friction caused by the air drag. $\mu_{r}$ is the corresponding coefficient.

\subsection{Coupled Equations}

We summarize the previous results and present the whole set of equations. We start with four abbreviations:

The moment of inertia of the permanent magnet is

$$
J=l \pi \rho \frac{a^{4}}{2} .
$$

The limit of the average temperature in the permanent magnet is

$$
\bar{T}^{\infty}=\frac{1}{2 \pi} \int_{0}^{2 \pi} T_{\mathrm{sur}}(\varphi) d \varphi .
$$

The levitation force at the limit temperature is

$$
\bar{F}_{y}^{\infty}=l \pi a^{2} \rho g .
$$

The inhomogeneous magnetization of the permanent magnet at temperature $\bar{T}^{\infty}$ yields the center of magnetization (at angle $\varphi=0$ )

$$
x_{0}^{m}=\frac{\int_{A} x m_{z}^{\infty}(x, y) d A}{\int_{A} m_{z}^{\infty}(x, y) d A}, \quad y_{0}^{m}=\frac{\int_{A} y m_{z}^{\infty}(x, y) d A}{\int_{A} m_{z}^{\infty}(x, y) d A} .
$$

With this abbreviations we end up with

$$
\begin{gathered}
\rho c\left[\frac{\partial T}{\partial t}+\omega\left(\begin{array}{c}
-y \\
x
\end{array}\right) \cdot \nabla T\right]-\nabla \cdot(\lambda \nabla T)=0 \text { in } A \\
\mathbf{n} \cdot(\lambda \nabla T)=\alpha\left(T_{\mathrm{Sur}}-T\right) \quad \text { on } \partial A \\
M_{\varphi}=\bar{F}_{y}^{\infty}\left[x_{0}^{m} \cos \varphi-y_{0}^{m} \sin \varphi\right] \\
M_{T}=\bar{F}_{y}^{\infty} \gamma \frac{1}{\pi a^{2}} \int_{A} x T(x, y) d A \\
\omega=\frac{\partial \varphi}{\partial t} \\
J \frac{\partial \omega}{\partial t}+\mu_{r} \omega=M_{\varphi}+M_{T}
\end{gathered}
$$




\section{Idealizations}

When performing the described experiment, it can be observed, that different permanent magnets show different behaviors, and also that the temperature plays an important role. Typically, the magnets start to oscillate around their horizontal axis (the $z$-axis in our model), and the amplitude increases. Some magnets then turn over and rotate continuously, other do not reach this state, and keep on with oscillations.

It is described in [5] that if the permanent magnet is first immersed in liquid nitrogen, then it will not rotate spontaneously, but any spin imparted on it is rapidly dissipated. In further observations it is described, that the angular speed of the magnet depends on changes on the temperature field: the angular speed is slowed down by pouring some cold nitrogen vapor over the magnet; or conversely, a speedup can be obtained when shining infrared light onto the magnet.

In the following, we will consider special cases of the set of partial differential equations (23)-(28), and explain what causes the different behaviors which are observed.

We start with a perfect magnet, and identify the reason for the continuous rotations. Then, we consider the special case of a homogeneous temperature field, explaining the observed oscillations. Later, in a subsequent chapter, we investigate the more realistic setting (an imperfect permanent magnet and a non-homogeneous temperature field), and present the results of finite element simulations of the coupled set of equations.

\subsection{The Perfect Permanent Magnet}

We call a permanent magnet perfect, if the center of its magnetization at temperature $\bar{T}^{\infty}$ is exactly the point $(0,0)$, i.e. in our notation

$$
\int_{A} x m_{z}^{\infty}(x, y) d A=\int_{A} y m_{z}^{\infty}(x, y) d A=0
$$

so that $x_{0}^{m}=y_{0}^{m}=0$.

Assuming a perfect permanent magnet, we see that $M_{\varphi}=0$ (cf. equation (25)). Thus, the driving torque is only $M_{T}$ (the temperature dependent torque). The model reduces immediately to $(23),(24),(26)$, and $(28)$ with $M_{\varphi}=0$. We will see, that this set of equations has solutions with a constant angular speed. Therefore, we look for stationary solutions of the equations. Setting the derivatives with respect to the time $t$ to zero, we arrive at

$$
\begin{gathered}
\rho c \omega\left(\begin{array}{c}
-y \\
x
\end{array}\right) \cdot \nabla T-\nabla \cdot(\lambda \nabla T)=0 \quad \text { in } A \\
\mathbf{n} \cdot(\lambda \nabla T)=\alpha\left(T_{\text {sur }}-T\right) \text { on } \partial A \\
M_{T}=\bar{F}_{y}^{\infty} \gamma \frac{1}{\pi a^{2}} \int_{A} x T(x, y) d A \\
\mu_{r} \omega=M_{T} .
\end{gathered}
$$


The meaning of the equations is as follows: The first two equations describe a stationary heat transfer problem with convection due to a constant angular speed $\omega$. The solution of these equations is a temperature field $T(x, y)$, which produces by (31) a torque $M_{T}$, which has to be as large as the damping by the stokes friction $\mu_{r} \omega$. So we end up with the situation of a rotating permanent magnet with constant angular speed $\omega$.

To simplify the forthcoming notations, we introduce the abbreviations $k=\lambda /(\rho c)$ and $h=\lambda / \alpha$, which transform equations (29) and (30) into

$$
\begin{gathered}
\omega\left(\begin{array}{c}
-y \\
x
\end{array}\right) \cdot \nabla T-k \Delta T=0 \text { in } A \\
h \frac{\partial T}{\partial \mathbf{n}}+T=T_{\text {sur }} \quad \text { on } \partial A
\end{gathered}
$$

We see from (31) that the driving torque $M_{T}$ is proportional to $\int_{A} x T(x, y) d A$, which, since $T$ is a solution of (33)-(34), is in turn dependent on the angular speed $\omega$. The crucial point for the whole process is the factor

$$
t x_{0}=\int_{A} x T(x, y) d A
$$

Therefore we are interested to study $x_{0}$ and its dependency on $\omega$. It is of advantage to consider $x_{0}$ in a complex setting. We define $\left(i^{2}=-1\right)$

$$
x_{0}=\int_{A} x T d A, \quad y_{0}=\int_{A} y T d A, \quad z_{0}=x_{0}+i y_{0}=\int_{A}(x+i y) T d A .
$$

Thus, the point $z_{0}$ in the complex plane is up to a constant factor the center of the temperature distribution in $A$.

We start with the case $\omega=0$ :

Theorem 3.1. Let $T$ be a solution of the stationary problem (33) and (34) with $\omega=0$. Then

$$
z_{0}=\frac{1}{4} \frac{a^{4}}{h+a} \int_{0}^{2 \pi} T_{s u r}(\varphi) e^{i \varphi} d \varphi
$$

Proof. If $\omega=0$, the solution $T$ is harmonic in $A$, so that we can write $T$ in polar coordinates as follows:

$$
T(r, \varphi)=C_{0}+\sum_{n=1}^{\infty} r^{n}\left[A_{n} \cos n \varphi+B_{n} \sin n \varphi\right] .
$$

Since

$$
z_{0}=\int_{0}^{a} \int_{0}^{2 \pi} r e^{i \varphi} T(r, \varphi) d \varphi r d r
$$


it follows with (36)

$$
z_{0}=\int_{0}^{a} r^{3} \int_{0}^{2 \pi} e^{i \varphi}\left[A_{1} \cos \varphi+B_{1} \sin \varphi\right] d \varphi d r=\frac{\pi}{4} a^{4}\left(A_{1}+i B_{1}\right) .
$$

Using (34) we get

$$
h A_{1}+a A_{1}=\frac{1}{\pi} \int_{0}^{2 \pi} T_{\mathrm{sur}}(\varphi) \cos \varphi d \varphi
$$

and a similar expression holds for $B_{1}$. Thus, it follows

$$
z_{0}=\frac{1}{4} \frac{a^{4}}{h+a} \int_{0}^{2 \pi} T_{\mathrm{Sur}}(\varphi) e^{i \varphi} d \varphi
$$

as required.

If the permanent magnet is rotating, i.e. $\omega \neq 0$, we have

Theorem 3.2. Let $T$ be a solution of the stationary problem (33) and (34) with $\omega \neq 0$. Then

$$
z_{0}=\frac{a^{2} J_{2}\left(\sqrt{\frac{\omega}{i k}} a\right)}{\frac{\omega}{i k} h J_{1}^{\prime}\left(\sqrt{\frac{\omega}{i k}} a\right)+\sqrt{\frac{\omega}{i k}} J_{1}\left(\sqrt{\frac{\omega}{i k}} a\right)} \int_{0}^{2 \pi} e^{i \varphi} T_{s u r}(\varphi) d \varphi,
$$

where $J_{\nu}$ is the Bessel function of first kind, i.e. (cf. [6])

$$
J_{\nu}(z)=\sum_{m=0}^{\infty} \frac{(-1)^{m} z^{\nu+2 m}}{m ! 2^{\nu+2 m} \Gamma(\nu+m+1)} .
$$

Proof. In polar coordinates, we can write

$$
z_{0}=\int_{0}^{a} r^{2} \sigma(r) d r
$$

where

$$
\sigma(r)=\int_{0}^{2 \pi} e^{i \varphi} T(r, \varphi) d \varphi
$$

Multiplying the stationary version of (6) with $e^{i \varphi}$ and integrating, we obtain

$$
\omega \int_{0}^{2 \pi} e^{i \varphi} \frac{\partial T}{\partial \varphi} d \varphi-k\left[\frac{\partial^{2}}{\partial r^{2}}+\frac{1}{r} \frac{\partial}{\partial r}\right] \int_{0}^{2 \pi} e^{i \varphi} T d \varphi-\frac{k}{r^{2}} \int_{0}^{2 \pi} e^{i \varphi} \frac{\partial^{2}}{\partial \varphi^{2}} T d \varphi=0 .
$$

Integration by parts yields

$$
\left[r^{2} \frac{\partial^{2}}{\partial r^{2}}+r \frac{\partial}{\partial r}\right] \sigma(r)-\left(\frac{\omega r^{2}}{i k}-1\right) \sigma(r)=0,
$$


since the integrals with partial derivatives with respect to $\varphi$ vanish. The change of variables

$$
\sqrt{\frac{i k}{\omega}} \rho=r, \quad \tilde{\sigma}(\rho)=\sigma\left(\sqrt{\frac{i k}{\omega} \rho}\right)
$$

yields

$$
\left[\rho^{2} \frac{\partial^{2}}{\partial \rho^{2}}+\rho \frac{\partial}{\partial \rho}\right] \tilde{\sigma}(\rho)+\left(\rho^{2}-1\right) \tilde{\sigma}(\rho)=0
$$

which is recognized as Bessel's differential equation (cf. [6]). Since we require that the solution is finite for $\rho=0$, the general solution is given by the Bessel function $J_{1}$, i.e.

$$
\tilde{\sigma}(\rho)=C J_{1}(\rho) .
$$

The constant $C$ is determined with the boundary conditions: Multiplying (34) in polar coordinates by $e^{i \varphi}$ and integrating, we obtain

$$
h \frac{\partial \sigma(r)}{\partial r}+\left.\sigma(r)\right|_{r=a}=\int_{0}^{2 \pi} e^{i \varphi} T_{\operatorname{Sur}}(\varphi) d \varphi
$$

so that

$$
C\left[h \sqrt{\frac{\omega}{i k}} J_{1}^{\prime}\left(\sqrt{\frac{\omega}{i k}} a\right)+J_{1}\left(\sqrt{\frac{\omega}{i k}} a\right)\right]=\int_{0}^{2 \pi} e^{i \varphi} T_{\operatorname{Sur}}(\varphi) d \varphi .
$$

In the next lemma we will show, that the expression in the brackets is not zero, so that we can solve the equation for $C$.

We finally arrive at

$$
\begin{aligned}
z_{0} & =\int_{0}^{a} r^{2} \sigma(r) d r \\
& =C \int_{0}^{a} r^{2} J_{1}\left(\sqrt{\frac{\omega}{i k}} r\right) d r \\
& =C \int_{0}^{\sqrt{\frac{\omega}{i k}} a} \rho^{2}\left(\frac{i k}{\omega}\right)^{3 / 2} J_{1}(\rho) d \rho \\
& =C\left(\frac{i k}{\omega}\right)^{1 / 2} a^{2} J_{2}\left(\sqrt{\frac{\omega}{i k}} a\right)
\end{aligned}
$$

which completes the proof in view of (38). The last equality is due to the integral formula $\int z^{2} J_{1}(z) d z=z^{2} J_{2}(z)$, which can be found, e.g. in [6].

The following lemma shows, that equation (38) can be solved for $C$ :

Lemma 3.3. Let $k, h, a>0$. If $\omega \neq 0$, then

$$
h \sqrt{\frac{\omega}{i k}} J_{1}^{\prime}\left(\sqrt{\frac{\omega}{i k}} a\right)+J_{1}\left(\sqrt{\frac{\omega}{i k}} a\right) \neq 0 .
$$




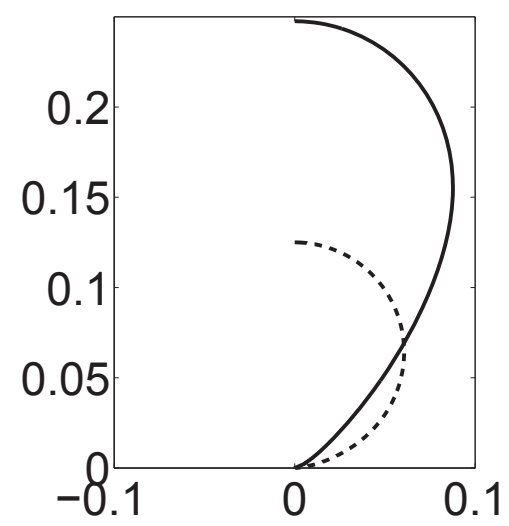

Figure 2: The center of temperature $i z_{0}(\omega)$ for $\omega \in[0, \infty)$. The parameters are $a=1$, $k=1$ and $h=0.01$, resp. $h=1$ (dashed line). $z_{0}$ is multiplied by $i$ to give the imagination of the experimental setup, where $T_{\text {Sur }}$ is dependent only on $y$ with a gradient pointing in $y$-direction.

Proof. It is known (cf. e.g. [6]), that if $A$ and $B$ are nonzero and real, the expression $A J_{\nu}(z)+B z J_{\nu}^{\prime}(z)$ has only real zeros except that it has two purely imaginary zeros when $\nu+A / B<0$. Multiplying (39) by $a$, it becomes obvious that the expression is nonzero for $\omega \neq 0$ since the arguments of the Bessel functions have both nonzero real and imaginary parts.

It convenient to have the result of the last theorem without derivatives of the Bessel function. This can be achieved using the recurrence relation $J_{2}(z)=J_{1}(z) / z-J_{1}^{\prime}(z)$ (cf. [6]). We get

Corollary 3.4. Under the assumptions of the last theorem, it holds

$$
z_{0}=\frac{a^{2} J_{2}\left(\sqrt{\frac{\omega}{i k}} a\right)}{\sqrt{\frac{\omega}{i k}}\left(1+\frac{h}{a}\right) J_{1}\left(\sqrt{\frac{\omega}{i k}} a\right)-\frac{\omega}{i k} h J_{2}\left(\sqrt{\frac{\omega}{i k}} a\right)} \int_{0}^{2 \pi} e^{i \varphi} T_{s u r}(\varphi) d \varphi .
$$

Some examples for $z_{0}=z_{0}(\omega)$ are given in Figure 2 .

It is interesting, that Theorem 3.1 can be seen as the limit case of the last corollary. Using well-known results for the Bessel functions, it is easy to prove

Theorem 3.5. Let $a, h$ and $k$ be positive. Then

$$
\lim _{\omega \rightarrow 0} \frac{a^{2} J_{2}\left(\sqrt{\frac{\omega}{i k}} a\right)}{\sqrt{\frac{\omega}{i k}}\left(1+\frac{h}{a}\right) J_{1}\left(\sqrt{\frac{\omega}{i k}} a\right)-\frac{\omega}{i k} h J_{2}\left(\sqrt{\frac{\omega}{i k}} a\right)}=\frac{1}{4} \frac{a^{4}}{h+a}
$$


and

$$
\lim _{\omega \rightarrow \infty} \frac{a^{2} J_{2}\left(\sqrt{\frac{\omega}{i k}} a\right)}{\sqrt{\frac{\omega}{i k}}\left(1+\frac{h}{a}\right) J_{1}\left(\sqrt{\frac{\omega}{i k}} a\right)-\frac{\omega}{i k} h J_{2}\left(\sqrt{\frac{\omega}{i k}} a\right)}=0
$$

Let us summarize the results for the perfect permanent magnet from a physical point of view:

(i) If the permanent magnet is rotating with the angular velocity $\omega$, a mechanical torque is produced, when there is a temperature gradient of the surrounding air in vertical direction. The torque has the same orientation as the rotational speed. The dependence of $M_{T}(\omega)$ on the temperature field is only via the moments $\int_{A} x T d A$ of the temperature field.

(ii) In a stationary situation, $\int_{A} x T d A$ can be calculated as follows. There is one multiplicative factor, which depends only on $\omega$. This dependency is explicitly expressible in terms of Bessel functions. The remaining factor depends only on the first Fourier coefficient of the temperature of the surrounding air. In particular (cf. Theorem 3.2)

$$
\int_{A} x T d A=\operatorname{Re}\left(\frac{a^{2} J_{2}\left(\sqrt{\frac{\omega}{i k}} a\right)}{\frac{\omega}{i k} h J_{1}^{\prime}\left(\sqrt{\frac{\omega}{i k}} a\right)+\sqrt{\frac{\omega}{i k}} J_{1}\left(\sqrt{\frac{\omega}{i k}} a\right)} \int_{0}^{2 \pi} e^{i \varphi} T_{\operatorname{Sur}}(\varphi) d \varphi\right)
$$

(iii) Again we assume a stationary solution with constant $\omega$. If $T_{\text {sur }}$ depends only on $y$ (as in the experiment), then $\operatorname{Re}\left(\int_{0}^{2 \pi} e^{i \varphi} T_{\mathrm{Sur}}(\varphi) d \varphi\right)=0$, so that $M_{T}(0)=0$, cf. Theorem 3.1.

(iv) Theorem 3.5 shows for the stationary case, that $\lim _{\omega \rightarrow \infty} M_{T}(\omega)=0$.

(v) Rotations with a fixed angular speed are possible, if the driving torque due to $\omega$ is as large as the resistance by the air drag. Thus, the condition for a continuous rotation with constant angular velocity is $M_{T}(\omega)=\mu_{r} \omega$.

\subsection{The Homogeneous Temperature Field}

Now we consider the case, when the temperature field around the permanent magnet (we drop the assumption of a perfect permanent magnet) is homogeneous, i.e. $T_{\text {sur }}=$ const. In this situation, the temperature $T(x, y)$ in $A$ will converge to the constant temperature $T(x, y)=T_{\text {sur }}=\bar{T}^{\infty}$. What happens with the equations (23)-(28) of our model?

Assuming that the relaxation of the temperature is so that temperature differences within the cylinder can be neglected, the heat transfer part of the equations can be ignored and in addition it holds $M_{T}=0$, since the temperature in $A$ is assumed to 
be constant. Thus, we end up with (26)-(28), which can be written in one equation as

$$
J \frac{\partial^{2} \varphi}{\partial t^{2}}+\mu_{r} \frac{\partial \varphi}{\partial t}=\bar{F}_{y}^{\infty}\left[x_{0}^{m} \cos \varphi-y_{0}^{m} \sin \varphi\right] .
$$

Remark 3.6. To simplify the forthcoming considerations, the notations are simplified in the following way: As described before, $\left(x_{0}^{m}, y_{0}^{m}\right)$ denotes the center of magnetization of the $\operatorname{disk} A$. We rotate the reference system of $A$ so that $x_{0}^{m}=0$ and $y_{0}^{m} \geq 0$. Furthermore, we introduce the abbreviations

$$
p=\frac{\bar{F}_{y}^{\infty} y_{0}^{m}}{J}, \quad q=\frac{\mu_{r}}{J}
$$

Then, the dynamical behavior of (40) is given by

$$
\ddot{\varphi}+q \dot{\varphi}+p \sin \varphi=0
$$

The parameter $q$ comes from the damping via the Stokes friction and is assumed to be positive. $p$ describes the eccentricity of the center of magnetization. Our assumptions on $y_{0}^{m}$ yield $p \geq 0$.

It is known (cf. [13]), that (42) is the differential equation of the damped mathematical pendulum. We summarize the main results for this differential equation in the following

\section{Theorem 3.7.}

(i) Let $p>0$, i.e. the permanent is not perfect. Then equation (42) has two critical points $(\varphi, \dot{\varphi})=(0,0)$ and $(\varphi, \dot{\varphi})=(\pi, 0)$ (and others via $2 \pi$-periodicity). The first one is a positive attractor, the second one is a negative attractor.

(ii) Let $p=0$, i.e. the permanent magnet is perfect. Then the solution of (42) satisfies

$$
\dot{\varphi}(t)=\dot{\varphi}(0) e^{-t / q} .
$$

Proof. (42) can be written as system

$$
\begin{aligned}
& \dot{\varphi}=\omega \\
& \dot{\omega}=-p \varphi-q \omega+p(\varphi-\sin \varphi)
\end{aligned}
$$

Then the assertion follows for the critical point $(\varphi, \omega)=(0,0)$ with the PoincaréLyapunov-Theorem (cf. [13]). The result for the critical point $(\varphi, \omega)=(\pi, 0)$ follows similarly by the substitution $\varphi \rightarrow \varphi+\pi$. This yields part (i) of the Theorem. Part (ii) is a standard result for linear ordinary differential equations.

From the previous theorem we can deduce the following facts for our experimental setting under the assumption, that $T_{\text {Sur }}=$ const: 
(i) If the permanent magnet is perfect, i.e. $x_{0}^{m}=y_{0}^{m}=0$ (or in other words $p=0$, then any rotation is damped with $\lim _{t \rightarrow \infty} \omega(t)=0$. The magnet does not show any oscillations.

(ii) If $p>0$, i.e. there exists an eccentricity for the center of magnetization, then we expect that the permanent magnet reaches its stable critical point $(\varphi, \omega)=$ $(0,0)$. The system shows the same behavior as a damped mathematical pendulum. If the damping is not too large, then oscillations will occur, and the equilibrium state is reached in an oscillatory way.

\section{Simulations}

The last chapter has shown what are the reasons for the typical behavior of a levitated permanent magnet. In short, we have seen that the continuous rotations of the permanent magnet are due to temperature gradients of the surrounding air, whereas the oscillations are a result of inhomogeneities in the magnetization of the cylindrical magnet. In order to study the real situation we present results of simulations, which where performed with the finite element code Femlab.

We start with results for a perfect permanent magnet and then show the results for the real situation.

The parameters of the simulations are summarized in Table 1. For all values we use SI units.

\begin{tabular}{|l|l|}
\hline \multicolumn{2}{|c|}{ Geometry and mechanical properties } \\
\hline$g$ & $9.81 \mathrm{~m} / \mathrm{s}^{2}$ \\
$a$ & $0.003 \mathrm{~m}$ \\
$l$ & $0.005 \mathrm{~m}$ \\
$\rho$ & $8236 \mathrm{~kg} / \mathrm{m}^{3}$ \\
$\mu_{r}$ & $1.04625 \cdot 10^{-10} \mathrm{~N} \mathrm{~m} \mathrm{~s}$ \\
$J=l \pi \rho a^{4} / 2$ & \\
$\bar{F}_{y}^{\infty}=l \pi a^{2} \rho g$ & \\
\hline \multicolumn{2}{|c|}{ Heat Transfer } \\
\hline$c$ & $376 \mathrm{~J} /(\mathrm{kg} \mathrm{K})$ \\
$\lambda$ & $8 \mathrm{~W} /(\mathrm{m} \mathrm{K})$ \\
$\alpha$ & $50 \mathrm{~W} /\left(\mathrm{m}^{2} \mathrm{~K}\right)$ \\
\hline Temperature Dependence of Magnetization \\
\hline$\gamma$ & $-0.0014 / \mathrm{K}$ \\
\hline
\end{tabular}

Table 1: Parameters for the simulations 


\subsection{The Perfect Permanent Magnet}

We assume the permanent magnet to be perfect, i.e. the center of magnetization coincides with the $z$-axis $\left(x_{m}^{0}=y_{m}^{0}=0\right)$. We present the results of three simulations. We consider the temperature fields

$$
T_{\text {sur }}^{i}(x, y)=-30+\tau_{i} y \quad\left(\text { in }{ }^{\circ} \mathrm{C}, y \text { in } \mathrm{m}\right),
$$

where $\tau_{1}=7000, \tau_{2}=10000$ and $\tau_{3}=13000$ (in $[\mathrm{K} / \mathrm{m}]$ ). The case $\tau_{2}$ corresponds with our experimental setup. Using the equilibrium condition $M_{T}(\omega)=\mu_{r} \omega$ we can calculate with the results of Section 3.1 the angular velocities at the stationary solutions corresponding to the different temperature fields. We get $\omega_{1}=6.63075[1 / \mathrm{s}]$, $\omega_{2}=7.98862[1 / \mathrm{s}]$, and $\omega_{3}=9.15695[1 / \mathrm{s}]$. Figure 3 shows the angular velocities for the three situations. In every simulation, we start with the initial conditions $\omega(0)=0.01[1 / \mathrm{s}]$ (i.e. we give the permanent magnet a small initial velocity) and $T(t=0, x, y)=-30\left[{ }^{\circ} \mathrm{C}\right]$ within $A$.

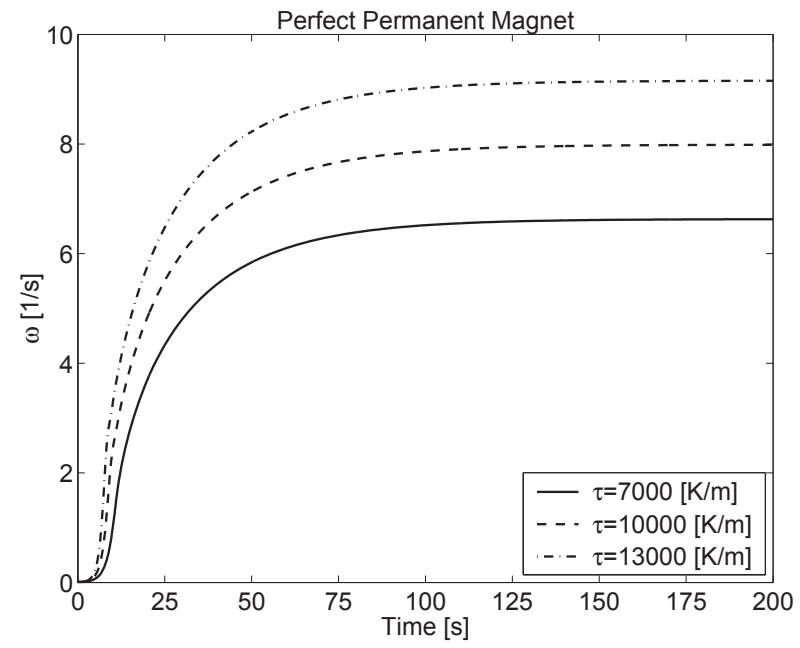

Figure 3: The Angular velocities for the perfect permanent magnet with three different surrounding temperatures $\left(T_{\mathrm{Sur}}^{i}, i=1,2,3\right)$.

Figure 4 gives a qualitative impression of the temperature field within the permanent magnet at a stationary solution.

\subsection{The Real Situation}

Now we assume that

$$
T_{\text {sur }}(x, y)=-30+10.000 y \quad\left(\text { in }{ }^{\circ} \mathrm{C}, y \text { in } \mathrm{m}\right),
$$




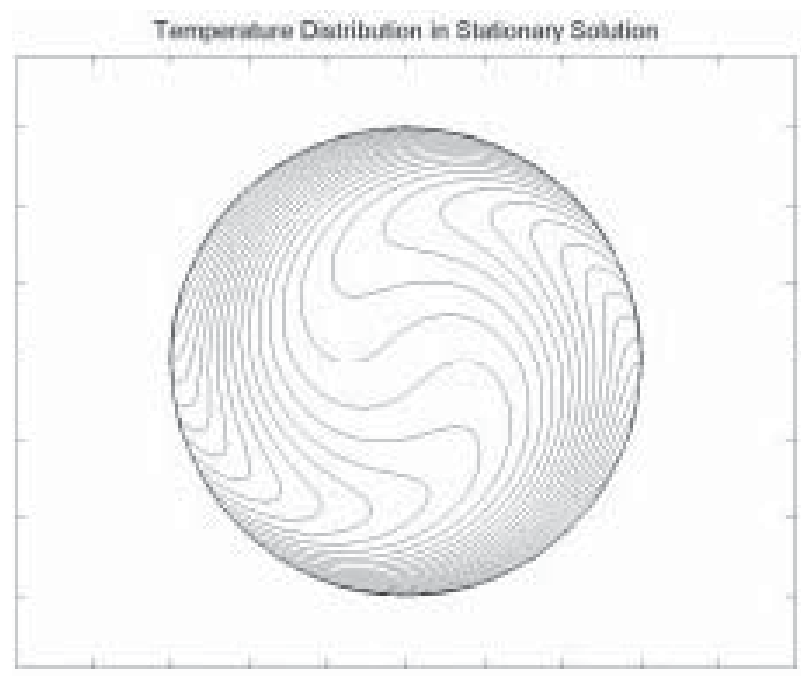

Figure 4: A typical temperature distribution within the rotating permanent magnet.

and show the results for the simulations with eccentricities $y_{0}^{m}=5 \cdot 10^{-7}[\mathrm{~m}], y_{0}^{m}=$ $10^{-6}[\mathrm{~m}]$, and $y_{0}^{m}=2.5 \cdot 10^{-6}[\mathrm{~m}]$. We see, that the typical behavior observed in experiments can be reproduced: the permanent magnet starts to oscillate, the amplitude increases, and a continuous rotation is reached. The initial conditions of these simulations are $\omega(0)=0.01[1 / \mathrm{s}], \varphi(0)=0$ and $T(t=0, x, y)=-30\left[{ }^{\circ} \mathrm{C}\right]$ within $A$. The figures 5,6 and 7 show the results for $\omega$. 


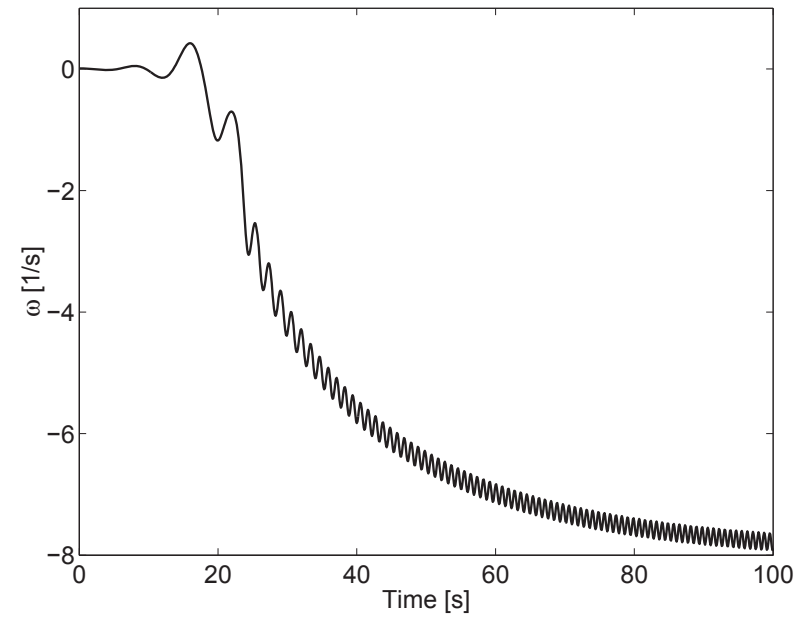

Figure 5: The angular velocity of a non-perfect permanent magnet in a nonhomogeneous temperature field. $y_{0}^{m}=5 \cdot 10^{-7}[\mathrm{~m}]$

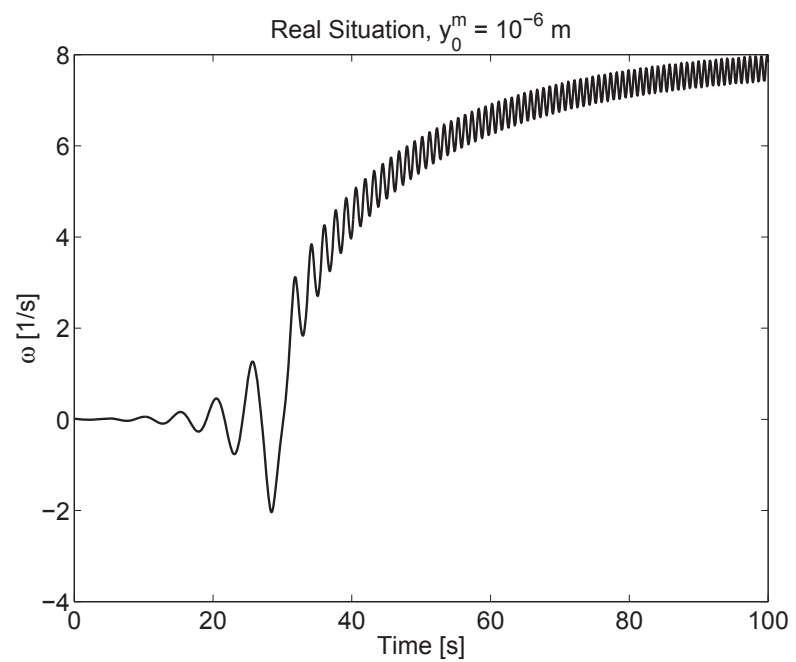

Figure 6: The angular velocity of a non-perfect permanent magnet in a nonhomogeneous temperature field. $y_{0}^{m}=\cdot 10^{-6}[\mathrm{~m}]$ 


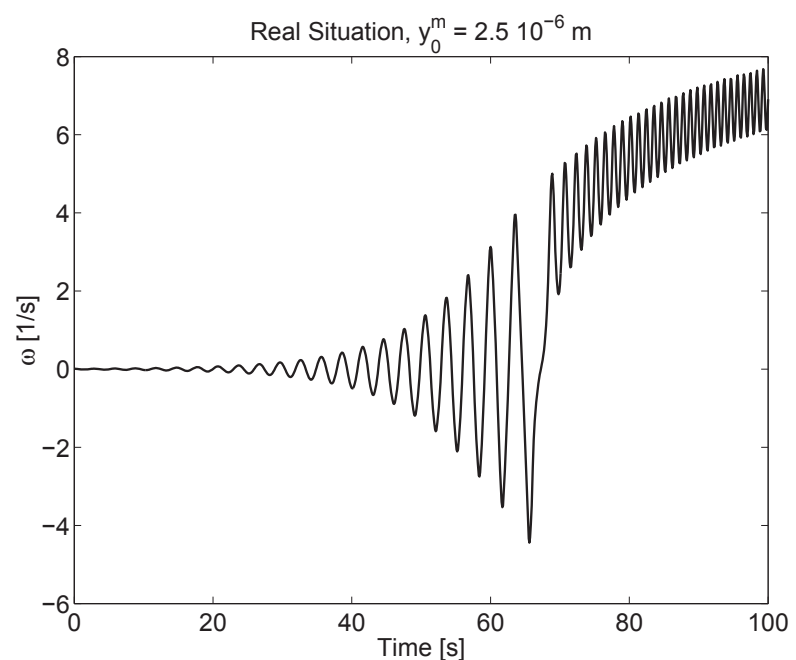

Figure 7: The angular velocity of a non-perfect permanent magnet in a nonhomogeneous temperature field. $y_{0}^{m}=2.5 \cdot 10^{-6}[\mathrm{~m}]$ 


\section{Conclusions}

It has been shown, that the model describes the behavior of a rotating permanent magnet, which is levitated above a superconducting disk. We have identified two sources for the rotation: $M_{T}$ and $M_{\varphi}$. The torque $M_{T}$, which is due to the temperature gradient of the surrounding air, depends on the angular speed $\omega$ of the magnet. Only when the center of magnetization of the permanent magnet does not coincide with the point $(0,0)$, there exits a restoring torque $M_{\varphi} . M_{\varphi}$ depends on the angular position of the permanent magnet $\varphi$. When both effects come together, the typical rotations of the permanent magnet are a consequence.

\section{Acknowledgements}

The stimulating discussions with my colleagues Prof. Dr. Claudio Palmy and Prof. Roland Rebsamen are greatfully acknowledged. The experiments performed by Claudio Palmy gave valuable insights in the mechanism of the rotating permanent magnet. I also want to thank Dr. Bernhard Fluche from the Femlab GmbH for the possibility to use a trial version of Femlab (developed by Comsol AB, Stockholm) for the finite element simulations.

\section{References}

[1] Arkadiev V. A Floating Magnet. Nature. 1947; 160: 330-330.

[2] Hellmann F, Gyorgy EM, Johnson DW, O'Bryan HM, Sherwood RC. Levitation of a magnet over a flat type II superconductor. Journal of Applied Physics. 1988; 63:447450.

[3] Incropera FP, DeWitt, DP. Fundamentals of Heat and Mass Transfer. John Wiley \& Sons: New York, 1996.

[4] Kellog OD. Foundation of Potential Theory. Springer: Berlin, Heidelberg, New York, 1967.

[5] Ma K, Liu J, McMichael C, Bruce R, Mims D, Chu W. Spontaneous and persistent rotation of cylindrical megnets levitated by $\mathrm{Y}-\mathrm{Ba}-\mathrm{Cu}-\mathrm{O}$ superconductors. Journal of Applied Physics. 1991; 70(7): 3961-3963.

[6] Magnus W, Oberhettinger F, Soni RP. Formulas and Theorems for the Special Functions of Mathematical Physics. Springer: Berlin, Heidelberg, New York, 1966.

[7] Martini G, Rivetti A, Pavese F. A self rotating magnet levitation above a YBCO specimen. Adv. Cryogenic Engineering. 1990; 35, 639-646.

[8] Moon F. Magneto-Solid Mechanics. John Wiley \& Sons: New York, 1984.

[9] Moon F. Superconducting levitation : applications to bearings and magnetic transportation (with selected sections by Pei-Zen Chang). John Wiley \& Sons: New York, 1994.

[10] Palmy C, Fullemann F. Thermal induced rotation of a levitated permanent magnet above a superconducting disk. Helv. Phys. Acta. 1990; 63(6):805-806. 
[11] Schreiner M, Palmy C. Why does a cylindrical magnet rotate when levitated above a superconducting plate? American Journal of Physics, accepted for publication.

[12] Tveito A, Winther R. Introduction to Partial Differential Equations. Springer: New York, 1998.

[13] Verhulst F. Nonlinear Differential Equations and Dynamical Systems, second edition. Springer: Berlin, Heidelberg, New York, 2000. 